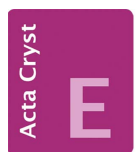

CRYSTALLOGRAPHIC COMMUNICATIONS

ISSN 2056-9890

Received 31 December 2016

Accepted 21 January 2017

Edited by A. Van der Lee, Université de Montpellier II, France

Keywords: crystal structure; powder diffraction; density functional theory; citrate; rubidium.

CCDC reference: 1529083

Supporting information: this article has supporting information at journals.iucr.org/e

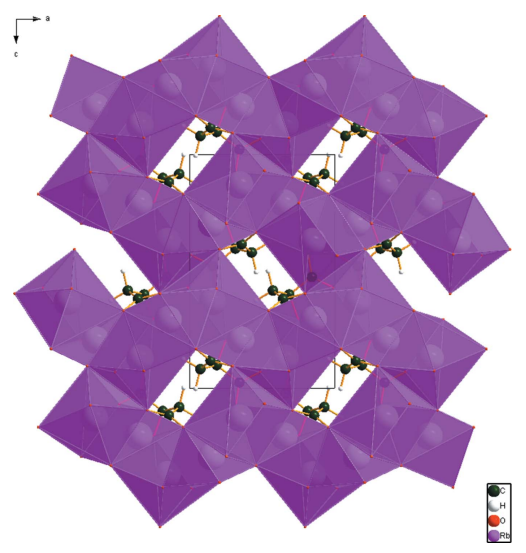

OPEN $\odot$ ACCESS

\section{Crystal structure of trirubidium citrate from laboratory X-ray powder diffraction data and DFT comparison}

\author{
Alagappa Rammohan ${ }^{\mathrm{a}}$ and James A. Kaduk ${ }^{\mathrm{b}}$ * \\ ${ }^{a}$ Atlantic International University, Honolulu HI, USA, and ${ }^{\mathbf{b}}$ Illinois Institute of Technology, Chicago IL, USA. \\ *Correspondence e-mail: kaduk@polycrystallography.com
}

The crystal structure of trirubidium citrate, $3 \mathrm{Rb}^{+} \cdot \mathrm{C}_{6} \mathrm{H}_{5} \mathrm{O}_{7}{ }^{3-}$, has been solved and refined using laboratory $\mathrm{X}$-ray powder diffraction data, and optimized using density functional techniques. The two independent $\mathrm{Rb}^{+}$cations are seven- and eight-coordinate, with bond-valence sums of 0.99 and 0.92 valence units. The coordination polyhedra share edges and corners to form a three-dimensional framework. The only hydrogen bond is an intramolecular one between the hydroxy group and the central carboxylate, with graph set $S(5)$. The hydrophobic methylene groups lie in pockets in the framework.

\section{Chemical context}

In the course of a systematic study of the crystal structures of Group 1 (alkali metal) citrate salts to understand the anion's conformational flexibility, ionization, coordination tendencies, and hydrogen bonding, we have determined several new crystal structures. Most of the new structures were solved using X-ray powder diffraction data (laboratory and/or synchrotron), but single crystals were used where available. The general trends and conclusions about the sixteen new compounds and twelve previously characterized structures are being reported separately (Rammohan \& Kaduk, 2017a). Eight of the new structures $-\mathrm{NaKHC}_{6} \mathrm{H}_{5} \mathrm{O}_{7}, \mathrm{NaK}_{2} \mathrm{C}_{6} \mathrm{H}_{5} \mathrm{O}_{7}$, $\mathrm{Na}_{3} \mathrm{C}_{6} \mathrm{H}_{5} \mathrm{O}_{7}, \quad \mathrm{NaH}_{2} \mathrm{C}_{6} \mathrm{H}_{5} \mathrm{O}_{7}, \quad \mathrm{Na}_{2} \mathrm{HC}_{6} \mathrm{H}_{5} \mathrm{O}_{7}, \quad \mathrm{~K}_{3} \mathrm{C}_{6} \mathrm{H}_{5} \mathrm{O}_{7}$, $\mathrm{Rb}_{2} \mathrm{HC}_{6} \mathrm{H}_{5} \mathrm{O}_{7}$, and $\mathrm{Rb}_{3} \mathrm{C}_{6} \mathrm{H}_{5} \mathrm{O}_{7}\left(\mathrm{H}_{2} \mathrm{O}\right)$ - have been published recently (Rammohan \& Kaduk, 2016a,b,c,d,e, 2017b,c; Rammohan et al., 2016), and two additional structures $\mathrm{KH}_{2} \mathrm{C}_{6} \mathrm{H}_{5} \mathrm{O}_{7}$ and $\mathrm{KH}_{2} \mathrm{C}_{6} \mathrm{H}_{5} \mathrm{O}_{7}\left(\mathrm{H}_{2} \mathrm{O}\right)_{2}$ - have been communicated to the CSD (Kaduk \& Stern, 2016a,b).

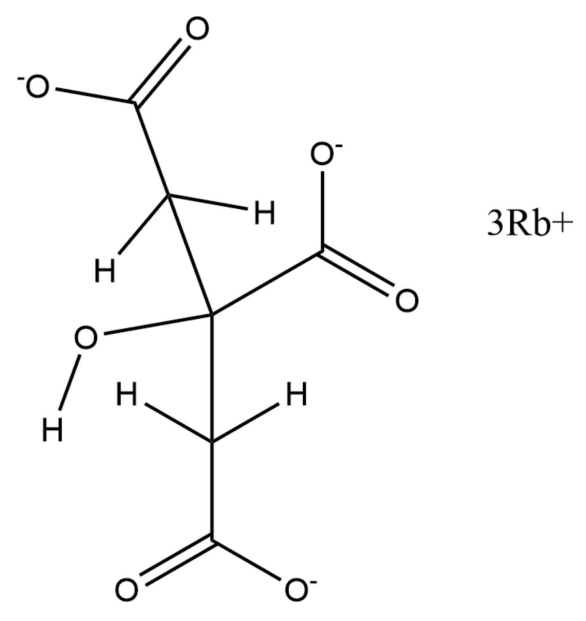




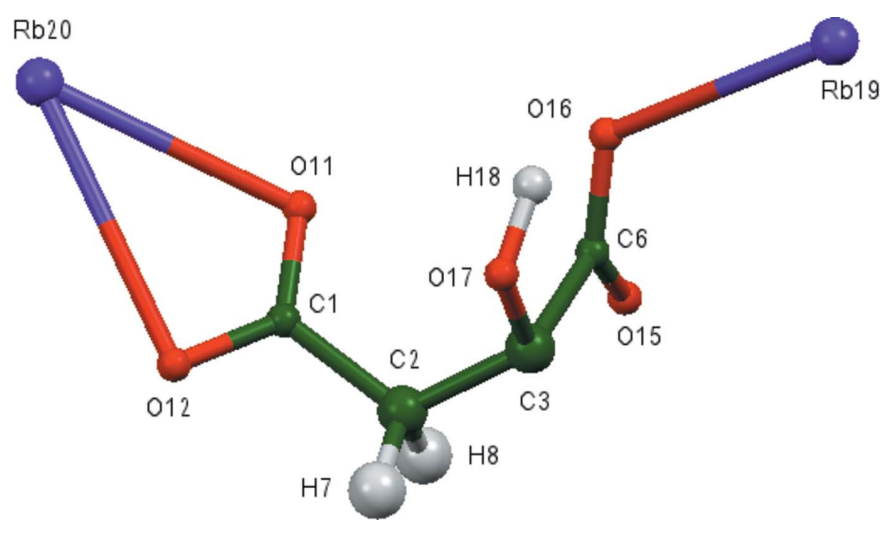

Figure 1

The asymmetric unit of trirubidium citrate, showing the atom numbering. The atoms are represented by $50 \%$ probability spheroids.

\section{Structural commentary}

The asymmetric unit of the title compound is shown in Fig. 1. The root-mean-square deviation of the non-hydrogen atoms in the Rietveld-refined and DFT-optimized structures is $0.052 \AA$ (Fig. 2). The largest difference is $0.086 \AA$, at $\mathrm{C} 1$. The excellent agreement between the two structures is strong evidence that the experimental structure is correct (van de Streek \& Neumann, 2014). This discussion uses the DFT-optimized structure. Most of the bond lengths, bond angles, and torsion angles fall within the normal ranges indicated by a Mercury Mogul geometry check (Macrae et al., 2008). The C3-C2-C1 angle of $116.5^{\circ}$ is flagged as unusual $[Z$-score $=2.3$; average $=$ $\left.112.7(16)^{\circ}\right]$. The $\mathrm{C} 2-\mathrm{C} 3-\mathrm{C} 2$ angle of $106.0^{\circ}$ is also flagged as unusual $\left[Z\right.$-score $=2.5$; average $\left.=109.6(14)^{\circ}\right]$. This hygroscopic compound was measured in situ, so perhaps slightly unusual geometry could be expected.

The citrate anion occurs in the trans,trans-conformation, which is one of the two low-energy conformations of an isolated citrate. The central carboxylate group and the hy-

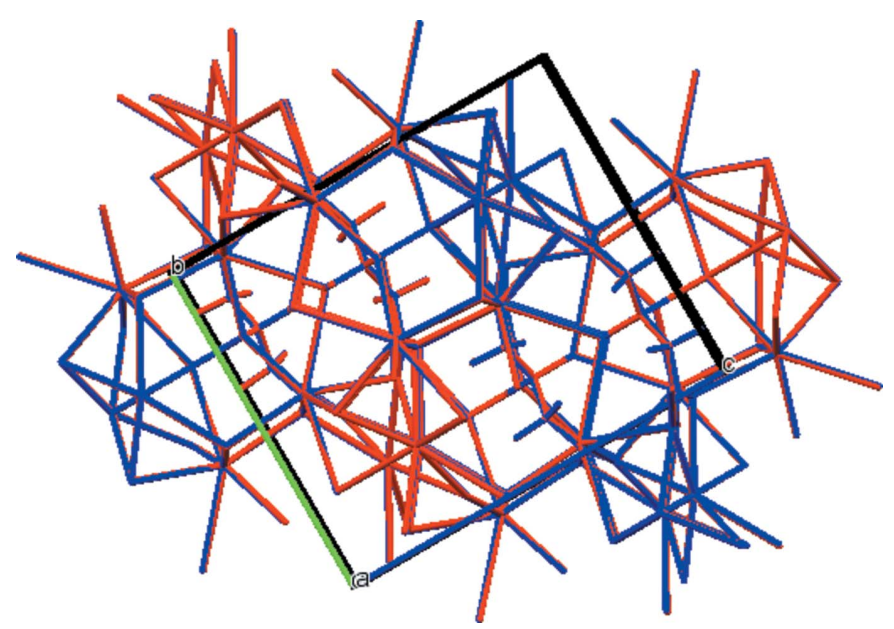

Figure 2

Comparison of the refined and optimized structures of trirubidium citrate. The refined structure is in red, and the DFT-optimized structure is in blue.
Table 1

Hydrogen-bond geometry $\left(\AA \stackrel{\circ}{\circ}^{\circ}\right)$ for ramm077c_DFT.

\begin{tabular}{lcccc}
\hline$D-\mathrm{H} \cdots A$ & $D-\mathrm{H}$ & $\mathrm{H} \cdots A$ & $D \cdots A$ & $D-\mathrm{H} \cdots A$ \\
\hline $\mathrm{O} 17-\mathrm{H} 18 \cdots \mathrm{O} 16$ & 0.981 & 1.862 & 2.572 & 126.7 \\
\hline
\end{tabular}

droxy group lie on a mirror plane. The terminal carboxylate O11 atom and the central carboxylate $\mathrm{O} 15$ atom chelate to $\mathrm{Rb} 19, \mathrm{O} 11$ and the central carboxylate $\mathrm{O} 16$ atom chelate to a second $\mathrm{Rb} 19$, and the terminal carboxylate $\mathrm{O} 12$ atom and the O17 hydroxy group chelate to a third Rb19. The terminal O11-C1-C12 carboxylate group acts as a bidentate ligand to $\mathrm{Rb} 20$. The Mulliken overlap populations and atomic charges indicate that the metal-oxygen bonding is ionic.

The Bravais-Friedel-Donnay-Harker (Bravais, 1866; Friedel, 1907; Donnay \& Harker, 1937) morphology suggests that we might expect blocky morphology for trirubidium citrate, with $\{011\}$ as the principal faces. A 4th-order spherical harmonic texture model was included in the refinement. The texture index was 1.001, indicating that preferred orientation was not significant for this rotated flat-plate specimen.

\section{Supramolecular features}

The two independent $\mathrm{Rb}^{+}$cations, $\mathrm{Rb} 19$ and $\mathrm{Rb} 20$, are sevenand eight-coordinate, with bond-valence sums of 0.99 and 0.92 valence units, respectively. The coordination polyhedra share edges and corners to form a three-dimensional network (Fig. 3). The only hydrogen bond is an intramolecular one (Table 1) between the hydroxy group and the central carboxylate, with graph set $S(5)$. The Mulliken overlap

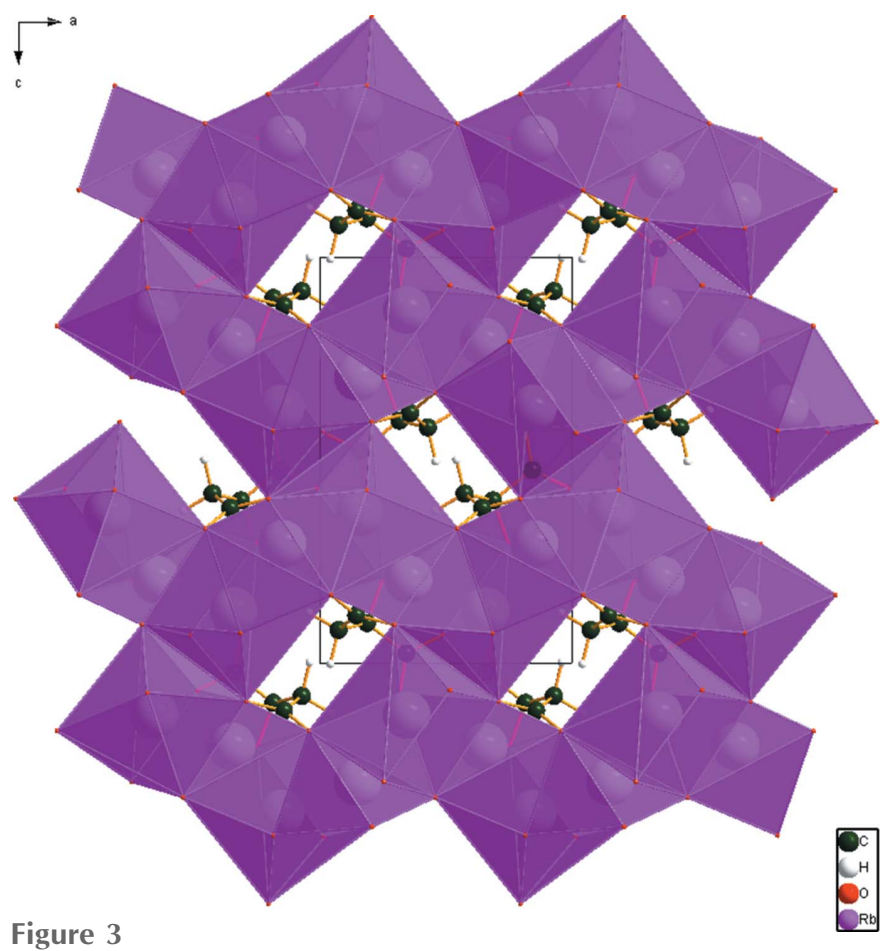

Crystal structure of trirubidium citrate, viewed down the $b$ axis. 


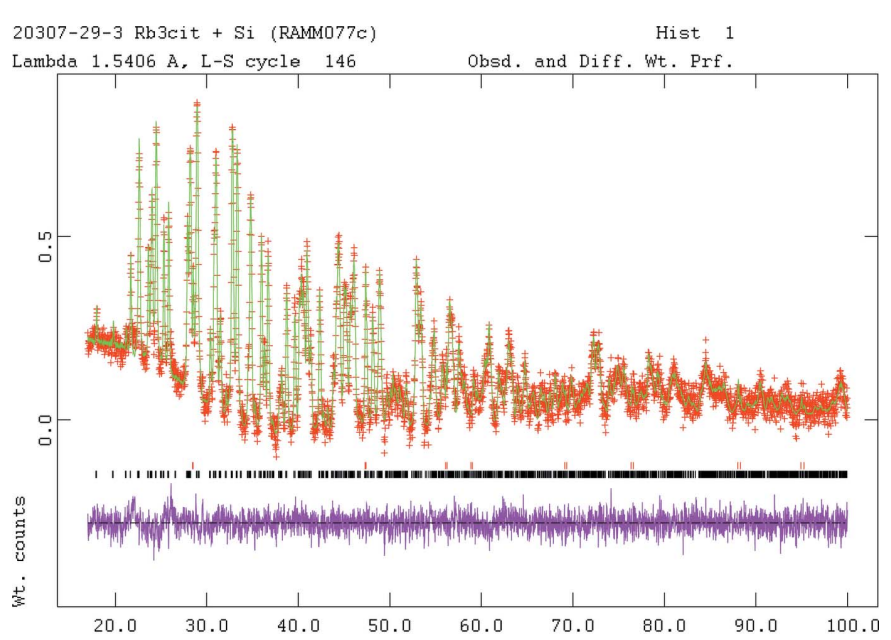

$2 \mathrm{gH}$, deg

Figure 4

Rietveld plot for the refinement of trirubidium citrate. The vertical scale is not the raw counts but the counts multiplied by the least-squares weights. This plot emphasizes the fit of the weaker peaks. The red crosses represent the observed data points, and the green line is the calculated pattern. The magenta curve is the difference pattern, plotted at the same scale as the other patterns. The row of black tick marks indicates the reflection positions. The red tick marks indicate the positions of the peaks of the Si internal standard.

population indicates, by the correlation in Rammohan \& Kaduk (2017a), that this hydrogen bond contributes $12.6 \mathrm{kcal}$ $\mathrm{mol}^{-1}$ to the crystal energy.

\section{Database survey}

Details of the comprehensive literature search for citrate structures are presented in Rammohan \& Kaduk (2017a). A reduced cell search of the cell of trirubidium citrate monohydrate in the Cambridge Structural Database (Groom et al., 2016) (increasing the default tolerance from 1.5 to $2.0 \%$ ) yielded 221 hits, but combining the cell search with the elements $\mathrm{C}, \mathrm{H}, \mathrm{O}$, and $\mathrm{Rb}$ only yielded no hits.

\section{Synthesis and crystallization}

A portion of $\mathrm{Rb}_{3}\left(\mathrm{C}_{6} \mathrm{H}_{5} \mathrm{O}_{7}\right)\left(\mathrm{H}_{2} \mathrm{O}\right)_{1}$ (Rammohan \& Kaduk, 2017c) was heated at $14 \mathrm{~K} \mathrm{~min}^{-1}$ to $463 \mathrm{~K}$ and held at that temperature for $10 \mathrm{~min}$. The white solid was immediately transferred to a glass vial to cool.

\section{Refinement}

Crystal data, data collection and structure refinement details are summarized in Table 2. Diffraction data are displayed in Fig. 4. The white solid was ground in a mortar and pestle, blended with NIST 640b Si internal standard in order to verify the calibrated goniometer zero error, packed into a standard Bruker D2 sample cell and protected from the atmosphere by an $8 \mu \mathrm{m}$ thick Kapton window attached to the cell with Vaseline. The powder pattern indicated that the sample was
Table 2

Experimental details.

\begin{tabular}{ll}
\hline Crystal data & \\
Chemical formula & $3 \mathrm{Rb}^{+} \cdot \mathrm{C}_{6} \mathrm{H}_{5} \mathrm{O}_{7}{ }^{3-}$ \\
$M_{\mathrm{r}}$ & 445.50 \\
Crystal system, space group & Orthorhombic, Pnma \\
Temperature (K) & 300 \\
$a, b, c(\AA)$ & $7.9096(2), 10.7733(3), 12.6986(3)$ \\
$V\left(\AA^{3}\right)$ & $1082.08(7)$ \\
$Z$ & 4 \\
Radiation type & $K \alpha_{1}, K \alpha_{2}, \lambda=1.540593,1.544451 \AA$ \\
Specimen shape, size $(\mathrm{mm})$ & Flat sheet, $24 \times 24$ \\
& \\
Data collection & Bruker D2 Phaser \\
Diffractometer & Standard PMMA holder with \\
Specimen mounting & Kapton window \\
& Reflection \\
Data collection mode & Step \\
Scan method & $2 \theta_{\min }=5.002 \theta_{\text {max }}=100.01$ \\
$2 \theta$ values $\left(^{\circ}\right)$ & $2 \theta_{\text {step }}=0.020$ \\
& \\
Refinement & $R_{\mathrm{p}}=0.020, R_{\mathrm{wp}}=0.025$, \\
$R$ factors and goodness of fit & $R_{\text {exp }}=0.023, R\left(F^{2}\right)=0.048$, \\
& $\chi^{2}=1.232$ \\
No. of parameters & 57 \\
No. of restraints & 13 \\
\hline
\end{tabular}

The same symmetry and lattice parameters were used for the DFT calculation. Computer programs: DIFFRAC.Measurement (Bruker, 2009), GSAS (Larson \& Von Dreele, 2004), DIAMOND (Crystal Impact, 2015) and publCIF (Westrip, 2010).

still hydrated, so the blend was re-heated at $17 \mathrm{~K} \mathrm{~min}^{-1}$ to 483 (10) $\mathrm{K}$ and held for $10 \mathrm{~min}$. Re-measuring the powder pattern indicated that a new phase had formed.

The pattern was indexed using DICVOL06 (Louër \& Boultif, 2007) on a primitive orthorhombic cell having $a=$ 7.904, $b=12.701, c=10.773 \AA$, and $V=1081.8 \AA^{3}$. These lattice parameters are $2.6,1.8$, and $3.3 \%$ larger than those of $\mathrm{K}_{3} \mathrm{C}_{6} \mathrm{H}_{5} \mathrm{O}_{7}$ (Rammohan \& Kaduk, 2016e), and the volume is $7.9 \%$ larger. The compound was assumed to be isostructural to the $\mathrm{K}$ analogue (space group $P n a 2_{1}$ ), and the coordinates of tripotassium citrate were used as the initial model for the Rietveld refinement.

Pseudo-Voigt profile coefficients were as parameterized in Thompson et al. (1987) with profile coefficients for Simpson's rule integration of the pseudo-Voigt function according to Howard (1982). The asymmetry correction of Finger et al. (1994) was applied, and microstrain broadening by Stephens (1999). The structure was refined by the Rietveld method using GSAS/EXPGUI (Larson \& Von Dreele, 2004; Toby, 2001). All $\mathrm{C}-\mathrm{C}$ and $\mathrm{C}-\mathrm{O}$ bond lengths were restrained, as were all bond angles. The hydrogen atoms were included at fixed positions, which were recalculated during the course of the refinement using Materials Studio (Dassault Systèmes, 2014). The $U_{\text {iso }}$ value of the $\mathrm{C}$ atom in the central part of the citrate anion, and the $\mathrm{C}$ and $\mathrm{O}$ atoms on the exterior, were constrained to be equal, and the $U_{\text {iso }}$ valuess of the hydrogen atoms were constrained to be 1.3 times those of the atoms to which they are attached.

The structure refined satisfactorily $\left(R_{\mathrm{wp}}=0.0301\right.$ and reduced $\chi^{2}=1.828$ for 69 variables) in space group $P n a 2_{1}$ (the space group of the $\mathrm{K}$ analogue), but both the ADDSYM 
module of PLATON (Spek, 2009) and the Find Symmetry module of Materials Studio (Dassault Systèmes, 2014) suggested the presence of an additional centre of symmetry, and that the space group was Pnma (with a transformation of axes). The tolerance on the search was $0.12 \AA$. Because lower residuals were obtained with fewer parameters, we believe that Pnma is the correct space group.

\section{DFT calculations}

After the Rietveld refinement, a density functional geometry optimization (fixed experimental unit cell) was carried out using CRYSTAL14 (Dovesi et al., 2014). The basis sets for the $\mathrm{C}, \mathrm{H}$, and $\mathrm{O}$ atoms were those of Peintinger et al. (2012), and the basis set for $\mathrm{Rb}$ was that of Schoenes et al. (2008). The calculation was run on eight $2.1 \mathrm{GHz}$ Xeon cores (each with 6 Gb RAM) of a 304-core Dell Linux cluster at IIT, used $8 k$ points and the B3LYP functional, and took about seven $h$. The $U_{\text {iso }}$ values from the Rietveld refinement were assigned to the optimized fractional coordinates.

\section{Acknowledgements}

We thank Andrey Rogachev for the use of computing resources at IIT.

\section{References}

Bravais, A. (1866). In Études Cristallographiques. Paris: Gauthier Villars.

Bruker (2009). DIFFRAC.Measurement. Bruker AXS Inc., Madison, Wisconsin, USA.

Crystal Impact (2015). DIAMOND. Crystal Impact GbR, Bonn, Germany. http://www.crystalimpact.com/diamond.

Dassault Systèmes (2014). Materials Studio. BIOVIA, San Diego California, USA.

Donnay, J. D. H. \& Harker, D. (1937). Am. Mineral. 22, 446-467.
Dovesi, R., Orlando, R., Erba, A., Zicovich-Wilson, C. M., Civalleri, B., Casassa, S., Maschio, L., Ferrabone, M., De La Pierre, M., D'Arco, P., Noël, Y., Causà, M., Rérat, M. \& Kirtman, B. (2014). Int. J. Quantum Chem. 114, 1287-1317.

Finger, L. W., Cox, D. E. \& Jephcoat, A. P. (1994). J. Appl. Cryst. 27, 892-900.

Friedel, G. (1907). Bull. Soc. Fr. Mineral. 30, 326-455.

Groom, C. R., Bruno, I. J., Lightfoot, M. P. \& Ward, S. C. (2016). Acta Cryst. B72, 171-179.

Howard, C. J. (1982). J. Appl. Cryst. 15, 615-620.

Kaduk, J. A. \& Stern, C. (2016a). CSD Communication 14464571446458. CCDC, Cambridge, England.

Kaduk, J. A. \& Stern, C. (2016b). CSD Communication 1446460 1446461. CCDC, Cambridge, England.

Larson, A. C. \& Von Dreele, R. B. (2004). General Structure Analysis System, (GSAS). Report LAUR, 86-784 Los Alamos National Laboratory, New Mexico, USA.

Louër, D. \& Boultif, A. (2007). Z. Kristallogr. Suppl. 2007, 191-196.

Macrae, C. F., Bruno, I. J., Chisholm, J. A., Edgington, P. R., McCabe, P., Pidcock, E., Rodriguez-Monge, L., Taylor, R., van de Streek, J. \& Wood, P. A. (2008). J. Appl. Cryst. 41, 466-470.

Peintinger, M. F., Oliveira, D. V. \& Bredow, T. (2012). J. Comput. Chem. 34, 451-459.

Rammohan, A. \& Kaduk, J. A. (2016a). Acta Cryst. E72, 170-173.

Rammohan, A. \& Kaduk, J. A. (2016b). Acta Cryst. E72, 403-406.

Rammohan, A. \& Kaduk, J. A. (2016c). Acta Cryst. E72, 793-796.

Rammohan, A. \& Kaduk, J. A. (2016d). Acta Cryst. E72, 854-857.

Rammohan, A. \& Kaduk, J. A. (2016e). Acta Cryst. E72, 1159-1162.

Rammohan, A. \& Kaduk, J. A. (2017a). Acta Cryst. B73. Submitted.

Rammohan, A. \& Kaduk, J. A. (2017b). Acta Cryst. E73, 92-95.

Rammohan, A. \& Kaduk, J. A. (2017c). Acta Cryst. E73, 286-290.

Rammohan, A., Sarjeant, A. A. \& Kaduk, J. A. (2016). Acta Cryst. E72, 943-946.

Schoenes, J., Racu, A.-M., Doll, K., Bukowski, Z. \& Karpinski, J. (2008). Phys. Rev. B, 77, 134515.

Spek, A. L. (2009). Acta Cryst. D65, 148-155.

Stephens, P. W. (1999). J. Appl. Cryst. 32, 281-289.

Streek, J. van de \& Neumann, M. A. (2014). Acta Cryst. B70, 10201032.

Thompson, P., Cox, D. E. \& Hastings, J. B. (1987). J. Appl. Cryst. 20, 79-83.

Toby, B. H. (2001). J. Appl. Cryst. 34, 210-213.

Westrip, S. P. (2010). J. Appl. Cryst. 43, 920-925. 


\section{supporting information}

Acta Cryst. (2017). E73, 250-253 [https://doi.org/10.1107/S2056989017001086]

Crystal structure of trirubidium citrate from laboratory X-ray powder diffraction data and DFT comparison

Alagappa Rammohan and James A. Kaduk

Computing details

Data collection: DIFFRAC.Measurement (Bruker, 2009) for RAMM077C_phase_1. Molecular graphics: DIAMOND (Crystal Impact, 2015) for RAMM077C_phase_1. Software used to prepare material for publication: publCIF (Westrip, 2010) for RAMM077C_phase_1.

(RAMM077C_phase_1) Trirubidium citrate

Crystal data

$3 \mathrm{Rb}^{+} \cdot \mathrm{C}_{6} \mathrm{H}_{5} \mathrm{O}_{7}^{3-}$

$M_{r}=445.50$

Orthorhombic, Pnma

Hall symbol: $-\mathrm{P} 2 \mathrm{ac} 2 \mathrm{n}$

$a=7.9096(2) \AA$

$b=10.7733(3) \AA$

$$
\begin{aligned}
& c=12.6986(3) \AA \\
& V=1082.08(7) \AA^{3} \\
& Z=4 \\
& D_{\mathrm{x}}=2.735 \mathrm{Mg} \mathrm{m}^{-3} \\
& T=300 \mathrm{~K}
\end{aligned}
$$

Fractional atomic coordinates and isotropic or equivalent isotropic displacement parameters $\left(\hat{A}^{2}\right)$

\begin{tabular}{lllll}
\hline & $x$ & $y$ & $z$ & $U_{\text {iso }}{ }^{*} / U_{\text {eq }}$ \\
\hline C1 & $0.8481(8)$ & $0.5192(4)$ & $0.1236(8)$ & $0.0129(18)^{*}$ \\
C2 & $0.9284(10)$ & $0.6373(4)$ & $0.0836(8)$ & $0.028(5)^{*}$ \\
C3 & $0.8129(13)$ & 0.75 & $0.1037(8)$ & $0.028(5)^{*}$ \\
C6 & $0.6630(17)$ & 0.75 & $0.0251(11)$ & $0.0129(18)^{*}$ \\
H7 & 1.03549 & 0.6517 & 0.12302 & $0.036(6)^{*}$ \\
H8 & 0.9452 & 0.62963 & -0.00257 & $0.036(6)^{*}$ \\
O11 & $0.6988(9)$ & $0.4914(7)$ & $0.0961(6)$ & $0.0129(18)^{*}$ \\
O12 & $0.9397(9)$ & $0.4386(5)$ & $0.1695(6)$ & $0.0129(18)^{*}$ \\
O15 & $0.6865(17)$ & 0.75 & $-0.0763(10)$ & $0.0129(18)^{*}$ \\
O16 & $0.5218(18)$ & 0.75 & $0.0658(10)$ & $0.0129(18)^{*}$ \\
O17 & $0.7506(12)$ & 0.75 & $0.2090(9)$ & $0.0129(18)^{*}$ \\
H18 & 0.61955 & 0.75 & 0.19313 & $0.017(2)^{*}$ \\
Rb19 & $0.3442(2)$ & $0.97103(16)$ & $0.12323(13)$ & $0.0255(6)^{*}$ \\
Rb20 & $0.1377(4)$ & 0.25 & $0.29027(16)$ & $0.0255(6)^{*}$ \\
\end{tabular}

Geometric parameters $\left(\AA,{ }^{\circ}\right)$

\begin{tabular}{llll}
\hline $\mathrm{C} 1-\mathrm{C} 2$ & $1.5102(11)$ & $\mathrm{O} 15-\mathrm{Rb} 20^{\text {viii }}$ & $3.074(13)$ \\
$\mathrm{C} 1-\mathrm{O} 11$ & $1.267(3)$ & $\mathrm{O} 15-\mathrm{Rb} 20^{\mathrm{ix}}$ & $3.053(14)$
\end{tabular}




\begin{tabular}{|c|c|c|c|}
\hline $\mathrm{C} 1-\mathrm{O} 12$ & $1.272(3)$ & $\mathrm{O} 16-\mathrm{C} 6$ & $1.231(19)$ \\
\hline $\mathrm{C} 2-\mathrm{C} 1$ & $1.5102(11)$ & O16-Rb19 & $2.859(8)$ \\
\hline $\mathrm{C} 2-\mathrm{C} 3$ & $1.5410(11)$ & $\mathrm{O} 16-\mathrm{Rb} 19^{\mathrm{i}}$ & $2.859(8)$ \\
\hline $\mathrm{C} 2-\mathrm{H} 7$ & $0.996(9)$ & $\mathrm{O} 16-\mathrm{Rb} 20^{\text {viii }}$ & 3.719 (13) \\
\hline $\mathrm{C} 2-\mathrm{H} 8$ & $1.105(10)$ & $\mathrm{O} 17-\mathrm{C} 3$ & $1.425(3)$ \\
\hline $\mathrm{C} 3-\mathrm{C} 2$ & $1.5410(11)$ & $\mathrm{O} 17-\mathrm{H} 18$ & $1.056(9)$ \\
\hline $\mathrm{C} 3-\mathrm{C} 2^{\mathrm{i}}$ & $1.5410(11)$ & $\mathrm{O} 17-\mathrm{Rb} 19^{\mathrm{ii}}$ & $3.280(8)$ \\
\hline $\mathrm{C} 3-\mathrm{C} 6$ & $1.5502(11)$ & $\mathrm{O} 17-\mathrm{Rb} 19^{\mathrm{x}}$ & $3.280(8)$ \\
\hline $\mathrm{C} 3-\mathrm{O} 17$ & $1.425(3)$ & $\mathrm{H} 18-\mathrm{O} 17$ & $1.056(9)$ \\
\hline $\mathrm{C} 6-\mathrm{C} 3$ & $1.5502(11)$ & $\mathrm{Rb} 19-\mathrm{O} 11^{\mathrm{i}}$ & $2.855(7)$ \\
\hline $\mathrm{C} 6-\mathrm{O} 15$ & $1.300(16)$ & $\mathrm{Rb} 19-\mathrm{O} 11^{\mathrm{xi}}$ & $3.766(7)$ \\
\hline $\mathrm{C} 6-\mathrm{O} 16$ & $1.231(19)$ & $\mathrm{Rb} 19-\mathrm{O} 11^{\mathrm{xii}}$ & $2.815(8)$ \\
\hline $\mathrm{H} 7-\mathrm{C} 2$ & $0.996(9)$ & $\mathrm{Rb} 19-\mathrm{O} 12^{\mathrm{xiii}}$ & $3.395(7)$ \\
\hline $\mathrm{H} 8-\mathrm{C} 2$ & $1.105(10)$ & $\mathrm{Rb} 19-\mathrm{O} 12^{\mathrm{xi}}$ & $2.906(7)$ \\
\hline $\mathrm{O} 11-\mathrm{C} 1$ & $1.267(3)$ & $\mathrm{Rb} 19-\mathrm{O} 15^{\mathrm{vii}}$ & $3.074(3)$ \\
\hline $\mathrm{O} 11-\mathrm{Rb} 19^{\mathrm{i}}$ & $2.855(7)$ & $\mathrm{Rb} 19-\mathrm{O} 16$ & $2.859(8)$ \\
\hline $\mathrm{O} 11-\mathrm{Rb} 19^{\mathrm{ii}}$ & $3.766(7)$ & $\mathrm{Rb} 19-\mathrm{O} 17^{\mathrm{xi}}$ & $3.280(8)$ \\
\hline $\mathrm{O} 11-\mathrm{Rb} 19^{\mathrm{iii}}$ & $2.815(8)$ & $\mathrm{Rb} 20-\mathrm{O} 11^{\mathrm{xiv}}$ & $3.013(7)$ \\
\hline $\mathrm{O} 11-\mathrm{Rb} 20^{\mathrm{iv}}$ & $3.013(7)$ & $\mathrm{Rb} 20-\mathrm{O} 11^{\mathrm{xv}}$ & $3.013(7)$ \\
\hline $\mathrm{O} 12-\mathrm{C} 1$ & $1.272(3)$ & $\mathrm{Rb} 20-\mathrm{O} 12^{\mathrm{xvi}}$ & $2.989(6)$ \\
\hline $\mathrm{O} 12-\mathrm{Rb} 19^{\mathrm{v}}$ & $3.395(7)$ & $\mathrm{Rb} 20-\mathrm{O} 12^{\mathrm{xvii}}$ & $2.989(6)$ \\
\hline $\mathrm{O} 12-\mathrm{Rb} 19^{\mathrm{ii}}$ & $2.906(7)$ & $\mathrm{Rb} 20-\mathrm{O} 12^{\mathrm{xiv}}$ & $3.178(7)$ \\
\hline $\mathrm{O} 12-\mathrm{Rb} 20^{\mathrm{vi}}$ & $2.989(6)$ & $\mathrm{Rb} 20-\mathrm{O} 12^{\mathrm{xv}}$ & $3.178(7)$ \\
\hline $\mathrm{O} 12-\mathrm{Rb} 20^{\mathrm{iv}}$ & $3.178(7)$ & $\mathrm{Rb} 20-\mathrm{O} 15^{\text {xviii }}$ & $3.074(13)$ \\
\hline $\mathrm{O} 15-\mathrm{C} 6$ & $1.300(16)$ & $\mathrm{Rb} 20-\mathrm{O} 15^{\mathrm{ix}}$ & $3.053(14)$ \\
\hline $\mathrm{O} 15-\mathrm{Rb} 19^{\mathrm{vii}}$ & $3.074(3)$ & $\mathrm{Rb} 20-\mathrm{O} 16^{\mathrm{xvii}}$ & $3.719(13)$ \\
\hline $\mathrm{O} 15-\mathrm{Rb} 19^{\mathrm{iii}}$ & $3.074(3)$ & & \\
\hline $\mathrm{C} 2-\mathrm{C} 1-\mathrm{O} 11$ & $119.9(3)$ & $\mathrm{Rb} 19^{\mathrm{xix}}-\mathrm{O} 17-\mathrm{Rb} 19^{\mathrm{xxi}}$ & $93.1(3)$ \\
\hline $\mathrm{C} 2-\mathrm{C} 1-\mathrm{O} 12$ & $119.3(3)$ & 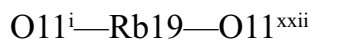 & $89.3(2)$ \\
\hline $\mathrm{C} 2-\mathrm{C} 1-\mathrm{Rb} 19^{\mathrm{xix}}$ & $108.2(5)$ & 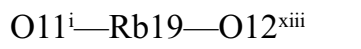 & $155.0(2)$ \\
\hline $\mathrm{O} 11-\mathrm{C} 1-\mathrm{O} 12$ & $119.7(3)$ & $\mathrm{O} 11^{\mathrm{i}}-\mathrm{Rb} 19-\mathrm{O} 12^{\mathrm{xxiii}}$ & $78.8(2)$ \\
\hline $\mathrm{C} 1-\mathrm{C} 2-\mathrm{C} 3$ & $111.0(3)$ & 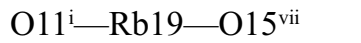 & $85.2(3)$ \\
\hline $\mathrm{C} 1-\mathrm{C} 2-\mathrm{H} 7$ & $108.7(5)$ & 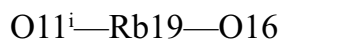 & $66.7(3)$ \\
\hline $\mathrm{C} 1-\mathrm{C} 2-\mathrm{H} 8$ & $108.7(7)$ & $\mathrm{O} 11^{\mathrm{i}}-\mathrm{Rb} 19-\mathrm{O} 17^{\mathrm{xiii}}$ & $113.8(3)$ \\
\hline $\mathrm{C} 3-\mathrm{C} 2-\mathrm{H} 7$ & $107.3(6)$ & 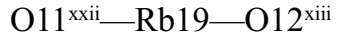 & $92.01(17)$ \\
\hline $\mathrm{C} 3-\mathrm{C} 2-\mathrm{H} 8$ & $107.1(5)$ & 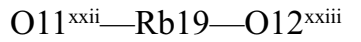 & $154.38(18)$ \\
\hline $\mathrm{H} 7-\mathrm{C} 2-\mathrm{H} 8$ & $114.0(8)$ & $\mathrm{O} 11^{\mathrm{xxii}}-\mathrm{Rb} 19-\mathrm{O} 15^{\mathrm{vii}}$ & $73.9(3)$ \\
\hline $\mathrm{C} 2-\mathrm{C} 3-\mathrm{C} 2^{\mathrm{i}}$ & $104.0(6)$ & $\mathrm{O} 11^{\mathrm{xxi}-\mathrm{Rb} 19-\mathrm{O} 16}$ & $82.6(3)$ \\
\hline $\mathrm{C} 2-\mathrm{C} 3-\mathrm{C} 6$ & $110.3(3)$ & O11 ${ }^{\mathrm{xxii}}-\mathrm{Rb} 19-\mathrm{O} 17^{\mathrm{xxiii}}$ & $132.2(2)$ \\
\hline $\mathrm{C} 2-\mathrm{C} 3-\mathrm{O} 17$ & $111.2(3)$ & $\mathrm{O} 12^{\mathrm{xii}}-\mathrm{Rb} 19-\mathrm{O} 12^{\mathrm{xxiii}}$ & $89.55(15)$ \\
\hline $\mathrm{C} 22^{\mathrm{i}}-\mathrm{C} 3-\mathrm{C} 6$ & $110.3(3)$ & $\mathrm{O} 12^{\mathrm{xiii}}-\mathrm{Rb} 19-\mathrm{O} 15^{\mathrm{vii}}$ & $71.3(3)$ \\
\hline $\mathrm{C} 2 \mathrm{i}-\mathrm{C} 3-\mathrm{O} 17$ & $111.2(3)$ & $\mathrm{O} 12^{\mathrm{xiii}}-\mathrm{Rb} 19-\mathrm{O} 16$ & $138.2(3)$ \\
\hline $\mathrm{C} 6-\mathrm{C} 3-\mathrm{O} 17$ & $109.9(3)$ & $\mathrm{O} 12^{\mathrm{xii}}-\mathrm{Rb} 19-\mathrm{O} 17^{\mathrm{xxiii}}$ & $83.3(2)$ \\
\hline $\mathrm{C} 3-\mathrm{C} 6-\mathrm{O} 15$ & $121.9(10)$ & $\mathrm{O} 12^{\mathrm{xxiii}}-\mathrm{Rb} 19-\mathrm{O} 15^{\mathrm{vii}}$ & $82.5(3)$ \\
\hline $\mathrm{C} 3-\mathrm{C} 6-\mathrm{O} 16$ & $115.1(10)$ & $\mathrm{O} 12^{\mathrm{xxiii}}-\mathrm{Rb} 19-\mathrm{O} 16$ & $112.5(3)$ \\
\hline $\mathrm{O} 15-\mathrm{C} 6-\mathrm{O} 16$ & $123.0(11)$ & $\mathrm{O} 12^{\mathrm{xxiii}}-\mathrm{Rb} 19-\mathrm{O} 17^{\mathrm{xxiii}}$ & $73.4(2)$ \\
\hline $\mathrm{C} 1-\mathrm{O} 11-\mathrm{Rb} 19^{\mathrm{i}}$ & $148.1(7)$ & 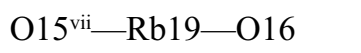 & $143.5(3)$ \\
\hline
\end{tabular}




\begin{tabular}{|c|c|c|c|}
\hline $\mathrm{C} 1-\mathrm{O} 11-\mathrm{Rb} 19^{\mathrm{xii}}$ & $113.8(6)$ & $\mathrm{O} 15^{\mathrm{vii}}-\mathrm{Rb} 19-\mathrm{O} 17^{\mathrm{xxiii}}$ & $144.9(3)$ \\
\hline $\mathrm{C} 1-\mathrm{O} 11-\mathrm{Rb} 20^{\mathrm{xx}}$ & $102.8(3)$ & $\mathrm{O} 16-\mathrm{Rb} 19-\mathrm{O} 17^{\mathrm{xxiii}}$ & $70.9(3)$ \\
\hline $\mathrm{Rb} 19^{\mathrm{i}}-\mathrm{O} 11-\mathrm{Rb} 19^{\mathrm{xii}}$ & $90.7(2)$ & $\mathrm{O} 11^{\mathrm{xxiv}}-\mathrm{Rb} 20-\mathrm{O} 11^{\mathrm{xxv}}$ & $119.3(3)$ \\
\hline $\mathrm{Rb} 19^{\mathrm{i}}-\mathrm{O} 11-\mathrm{Rb} 20^{\mathrm{xx}}$ & $84.7(2)$ & $\mathrm{O} 11^{\mathrm{xxiv}}-\mathrm{Rb} 20-\mathrm{O} 12^{\mathrm{xvi}}$ & $156.4(3)$ \\
\hline $\mathrm{Rb} 19^{\mathrm{xii}}-\mathrm{O} 11-\mathrm{Rb} 20^{\mathrm{xx}}$ & $112.8(3)$ & $\mathrm{O} 11^{\mathrm{xxiv}}-\mathrm{Rb} 20-\mathrm{O} 12^{\mathrm{xvii}}$ & $75.1(2)$ \\
\hline $\mathrm{C} 1-\mathrm{O} 12-\mathrm{Rb} 19^{\mathrm{v}}$ & $105.2(4)$ & $\mathrm{O} 11^{\mathrm{xxiv}}-\mathrm{Rb} 20-\mathrm{O} 12^{\mathrm{xxiv}}$ & $41.44(11)$ \\
\hline $\mathrm{C} 1-\mathrm{O} 12-\mathrm{Rb} 19^{\mathrm{xix}}$ & $92.2(6)$ & $\mathrm{O} 11^{\mathrm{xxiv}}-\mathrm{Rb} 20-\mathrm{O} 12^{\mathrm{xxv}}$ & $110.8(2)$ \\
\hline $\mathrm{C} 1-\mathrm{O} 12-\mathrm{Rb} 20^{\mathrm{vi}}$ & $175.9(6)$ & $\mathrm{O} 11^{\mathrm{xxiv}}-\mathrm{Rb} 20-\mathrm{O} 15^{\mathrm{xviii}}$ & $82.52(19)$ \\
\hline $\mathrm{C} 1-\mathrm{O} 12-\mathrm{Rb} 20^{\mathrm{xx}}$ & $94.7(3)$ & $\mathrm{O} 11^{\text {xxiv }}-\mathrm{Rb} 20-\mathrm{O} 15^{\mathrm{ix}}$ & $110.68(17)$ \\
\hline $\mathrm{Rb} 19^{\mathrm{v}}-\mathrm{O} 12-\mathrm{Rb} 19^{\mathrm{xix}}$ & $107.8(2)$ & $\mathrm{O} 11^{\mathrm{xxv}}-\mathrm{Rb} 20-\mathrm{O} 12^{\mathrm{xvi}}$ & $75.1(2)$ \\
\hline $\mathrm{Rb} 19^{\mathrm{v}}-\mathrm{O} 12-\mathrm{Rb} 20^{\mathrm{vi}}$ & $77.87(16)$ & $\mathrm{O} 11^{\mathrm{xxv}}-\mathrm{Rb} 20-\mathrm{O} 12^{\mathrm{xvii}}$ & $156.4(3)$ \\
\hline $\mathrm{Rb} 19^{\mathrm{v}}-\mathrm{O} 12-\mathrm{Rb} 20^{\mathrm{xx}}$ & $156.85(19)$ & $\mathrm{O} 11^{\mathrm{xxv}}-\mathrm{Rb} 20-\mathrm{O} 12^{\mathrm{xxiv}}$ & $110.8(2)$ \\
\hline $\mathrm{Rb} 19^{\mathrm{xix}}-\mathrm{O} 12-\mathrm{Rb} 20^{\mathrm{vi}}$ & $84.22(19)$ & $\mathrm{O} 11^{\mathrm{xxv}}-\mathrm{Rb} 20-\mathrm{O} 12^{\mathrm{xxv}}$ & $41.44(11)$ \\
\hline $\mathrm{Rb} 19^{\mathrm{xix}}-\mathrm{O} 12-\mathrm{Rb} 20^{\mathrm{xx}}$ & $82.72(18)$ & $\mathrm{O} 11^{\mathrm{xxv}}-\mathrm{Rb} 20-\mathrm{O} 15^{\mathrm{xviii}}$ & $82.52(19)$ \\
\hline $\mathrm{Rb} 20^{\mathrm{vi}}-\mathrm{O} 12-\mathrm{Rb} 20^{\mathrm{xx}}$ & $82.91(15)$ & $\mathrm{O} 11^{\mathrm{xx}}-\mathrm{Rb} 20-\mathrm{O} 15^{\mathrm{ix}}$ & $110.68(17)$ \\
\hline $\mathrm{C} 6-\mathrm{O} 15-\mathrm{Rb} 19^{\mathrm{vii}}$ & $100.4(3)$ & $\mathrm{O} 12^{\mathrm{xvi}}-\mathrm{Rb} 20-\mathrm{O} 12^{\mathrm{xvii}}$ & $85.7(3)$ \\
\hline $\mathrm{C} 6-\mathrm{O} 15-\mathrm{Rb} 19^{\mathrm{xii}}$ & $100.4(3)$ & $\mathrm{O} 12^{\mathrm{xvi}}-\mathrm{Rb} 20-\mathrm{O} 12^{\mathrm{xxiv}}$ & $155.67(15)$ \\
\hline $\mathrm{C} 6-\mathrm{O} 15-\mathrm{Rb} 20^{\mathrm{viii}}$ & $115.2(9)$ & $\mathrm{O} 12^{\mathrm{xvi}}-\mathrm{Rb} 20-\mathrm{O} 12^{\mathrm{xxv}}$ & $92.37(19)$ \\
\hline $\mathrm{C} 6-\mathrm{O} 15-\mathrm{Rb} 20^{\mathrm{ix}}$ & $161.1(10)$ & $\mathrm{O} 12^{\mathrm{xvi}}-\mathrm{Rb} 20-\mathrm{O} 15^{\mathrm{xvii}}$ & $81.1(2)$ \\
\hline $\mathrm{Rb} 19^{\mathrm{vii}}-\mathrm{O} 15-\mathrm{Rb} 19^{\mathrm{xii}}$ & $155.8(5)$ & $\mathrm{O} 12^{\mathrm{xvi}}-\mathrm{Rb} 20-\mathrm{O} 15^{\mathrm{ix}}$ & $77.4(2)$ \\
\hline $\mathrm{Rb} 19^{\text {vii }}-\mathrm{O} 15-\mathrm{Rb} 20^{\text {viii }}$ & $80.0(2)$ & $\mathrm{O} 12^{\mathrm{xvii}}-\mathrm{Rb} 20-\mathrm{O} 12^{\mathrm{xxiv}}$ & 92.37 (19) \\
\hline $\mathrm{Rb} 19^{\text {vii }}-\mathrm{O} 15-\mathrm{Rb} 20^{\mathrm{ix}}$ & $82.1(3)$ & $\mathrm{O} 12^{\mathrm{xvii}}-\mathrm{Rb} 20-\mathrm{O} 12^{\mathrm{xxv}}$ & $155.67(15)$ \\
\hline $\mathrm{Rb} 19^{\mathrm{xii}}-\mathrm{O} 15-\mathrm{Rb} 20^{\text {viii }}$ & $80.0(2)$ & $\mathrm{O} 12^{\mathrm{xvii}}-\mathrm{Rb} 20-\mathrm{O} 15^{\mathrm{xvii}}$ & $81.1(2)$ \\
\hline $\mathrm{Rb} 19^{\mathrm{xii}}-\mathrm{O} 15-\mathrm{Rb} 20^{\mathrm{ix}}$ & $82.1(3)$ & $\mathrm{O} 12^{\mathrm{xvi}}-\mathrm{Rb} 20-\mathrm{O} 15^{\mathrm{ix}}$ & $77.4(2)$ \\
\hline $\mathrm{Rb} 20^{\text {viii }}-\mathrm{O} 15-\mathrm{Rb} 20^{\mathrm{ix}}$ & $83.6(3)$ & $\mathrm{O} 12^{\mathrm{xxi}}-\mathrm{Rb} 20-\mathrm{O} 12^{\mathrm{xxv}}$ & $79.5(2)$ \\
\hline $\mathrm{C} 6-\mathrm{O} 16-\mathrm{Rb} 19$ & $123.6(2)$ & $\mathrm{O} 12^{\mathrm{xxiv}}-\mathrm{Rb} 20-\mathrm{O} 15^{\mathrm{xviii}}$ & $122.6(2)$ \\
\hline $\mathrm{C} 6-\mathrm{O} 16-\mathrm{Rb}^{1}{ }^{\mathrm{i}}$ & $123.6(2)$ & $\mathrm{O} 12^{\mathrm{xxi}}-\mathrm{Rb} 20-\mathrm{O} 15^{\mathrm{ix}}$ & $78.5(2)$ \\
\hline $\mathrm{Rb} 19-\mathrm{O} 16-\mathrm{Rb} 19^{\mathrm{i}}$ & $112.8(5)$ & $\mathrm{O} 12^{\mathrm{xx}}-\mathrm{Rb} 20-\mathrm{O} 15^{\mathrm{xviii}}$ & $122.6(2)$ \\
\hline $\mathrm{C} 3-\mathrm{O} 17-\mathrm{H} 18$ & $99.2(8)$ & $\mathrm{O} 12^{\mathrm{xx}}-\mathrm{Rb} 20-\mathrm{O} 15^{\mathrm{ix}}$ & $78.5(2)$ \\
\hline $\mathrm{C} 3-\mathrm{O} 17-\mathrm{Rb} 19^{\mathrm{xix}}$ & $122.1(3)$ & $\mathrm{O} 15^{\mathrm{xviii}}-\mathrm{Rb} 20-\mathrm{O} 15^{\mathrm{ix}}$ & $150.6(4)$ \\
\hline $\mathrm{C} 3-\mathrm{O} 17-\mathrm{Rb} 19^{\mathrm{xxi}}$ & $122.1(3)$ & & \\
\hline
\end{tabular}

Symmetry codes: (i) $x,-y+3 / 2, z$; (ii) $x+1 / 2,-y+3 / 2,-z+1 / 2$; (iii) $-x+1, y-1 / 2,-z$; (iv) $x+1 / 2,-y+1 / 2,-z+1 / 2$; (v) $x+1,-y+3 / 2, z$; (vi) $x+1, y, z$; (vii) $-x+1$, $-y+2,-z$; (viii) $-x+1 / 2, y+1 / 2, z-1 / 2$; (ix) $-x+1,-y+1,-z$; (x) $x+1 / 2, y,-z+1 / 2$; (xi) $x-1 / 2,-y+3 / 2,-z+1 / 2$; (xii) $-x+1, y+1 / 2,-z$; (xiii) $x-1,-y+3 / 2, z$; (xiv) $x-1 / 2,-y+1 / 2,-z+1 / 2$; (xv) $x-1 / 2, y,-z+1 / 2$; (xvi) $x-1, y, z$; (xvii) $x-1,-y+1 / 2, z$; (xviii) $-x+1 / 2, y-1 / 2, z+1 / 2$; (xix) $x+3 / 2,-y+5 / 2,-z+3 / 2$; (xx) $x+3 / 2,-y+3 / 2,-z+3 / 2$; (xxi) $x+3 / 2, y,-z+3 / 2$; (xxii) $-x+1, y+3 / 2,-z$; (xxiii) $x+1 / 2,-y+5 / 2,-z+3 / 2$; (xxiv) $x+1 / 2,-y+3 / 2,-z+3 / 2 ;(x x v) x+1 / 2, y,-z+3 / 2$.

(RAMM077C_phase_2)

Crystal data

$\mathrm{Si}$

$M_{r}=28.09$

Cubic, $F \overline{3} \overline{3} m$

Hall symbol: $-\mathrm{F} 4 \mathrm{vw} 2 \mathrm{vw}$

$a=5.43105 \AA$

$$
\begin{aligned}
& V=160.20 \AA^{3} \\
& Z=8 \\
& D_{\mathrm{x}}=2.329 \mathrm{Mg} \mathrm{m}^{-3} \\
& T=300 \mathrm{~K}
\end{aligned}
$$

Fractional atomic coordinates and isotropic or equivalent isotropic displacement parameters $\left(\AA^{2}\right)$

\begin{tabular}{lllll}
\hline & $x$ & $y$ & $z$ & $U_{\text {iso }} * / U_{\text {eq }}$ \\
\hline Si1 & 0.125 & 0.125 & 0.125 & $0.01 *$ \\
\hline
\end{tabular}


Geometric parameters $\left(\AA,{ }^{o}\right)$

\begin{tabular}{|c|c|c|c|}
\hline $\mathrm{Si} 1-\mathrm{Si}^{\mathrm{i}}$ & 2.3517 & $\mathrm{Si} 1-\mathrm{Si} 1^{\mathrm{iii}}$ & 2.3517 \\
\hline $\mathrm{Si} 1-\mathrm{Si}^{\mathrm{ii}}$ & 2.3517 & $\mathrm{Si} 1-\mathrm{Si}^{\mathrm{iv}}$ & 2.3517 \\
\hline $\mathrm{Si} 1^{\mathrm{i}}-\mathrm{Si} 1-\mathrm{Si} 1^{\mathrm{ii}}$ & 109.4712 & $\mathrm{Si} 1^{\mathrm{ii}}-\mathrm{Si} 1-\mathrm{Si} 1^{\mathrm{iii}}$ & 109.4712 \\
\hline $\mathrm{Si} 1^{\mathrm{i}}-\mathrm{Si} 1-\mathrm{Si} 1^{\mathrm{iii}}$ & 109.4712 & $\mathrm{Si} 1^{\mathrm{ii}}-\mathrm{Si} 1-\mathrm{Si}^{\mathrm{iv}}$ & 109.4712 \\
\hline $\mathrm{Si} 1^{\mathrm{i}}-\mathrm{Si} 1-\mathrm{Si} 1^{\mathrm{iv}}$ & 109.4712 & $\mathrm{Si} 1^{1 \mathrm{ii}}-\mathrm{Si} 1-\mathrm{Si} 1^{\mathrm{iv}}$ & 109.4712 \\
\hline
\end{tabular}

Symmetry codes: (i) $x+1 / 4, y+1 / 4,-z$; (ii) $-z, x+1 / 4, y+1 / 4$; (iii) $y+1 / 4,-z, x+1 / 4$; (iv) $-x,-y,-z$.

(ramm077c_DFT)

Crystal data

$\mathrm{C}_{6} \mathrm{H}_{5} \mathrm{O}_{7} \mathrm{Rb}_{3}$

$M_{r}=445.50$

$b=10.7733 \AA$

Orthorhombic, Pnma

$a=7.9096 \AA$

$c=12.6986 \AA$

$V=1082.08 \AA^{3}$

$Z=4$

Data collection

$h=\rightarrow$

$l=\rightarrow$

$k=\rightarrow$

Fractional atomic coordinates and isotropic or equivalent isotropic displacement parameters $\left(\AA^{2}\right)$

\begin{tabular}{lllll}
\hline & $x$ & $y$ & $z$ & $U_{\text {iso }}^{*} / U_{\text {eq }}$ \\
\hline C1 & 0.85652 & 0.51237 & 0.11762 & $0.01290^{*}$ \\
C2 & 0.92710 & 0.63666 & 0.08156 & $0.02780^{*}$ \\
H7 & 1.04404 & 0.65330 & 0.12425 & $0.03610^{*}$ \\
H8 & 0.95879 & 0.63156 & -0.00199 & $0.03610^{*}$ \\
O11 & 0.70573 & 0.48606 & 0.09469 & $0.01290^{*}$ \\
O12 & 0.95620 & 0.44175 & 0.16822 & $0.01290^{*}$ \\
Rb19 & 0.34329 & 0.97183 & 0.12807 & $0.02550^{*}$ \\
C3 & 0.81408 & 0.75000 & 0.09868 & $0.02780^{*}$ \\
C6 & 0.65846 & 0.75000 & 0.02369 & $0.01290^{*}$ \\
O15 & 0.68502 & 0.75000 & -0.07431 & $0.01290^{*}$ \\
O16 & 0.51559 & 0.75000 & 0.06782 & $0.01290^{*}$ \\
O17 & 0.75259 & 0.75000 & 0.20645 & $0.01290^{*}$ \\
H18 & 0.62975 & 0.75000 & 0.19609 & $0.01680^{*}$ \\
Rb20 & 0.14960 & 0.25000 & 0.29368 & $0.02550^{*}$ \\
\hline
\end{tabular}

Bond lengths (Å)

\begin{tabular}{llll}
\hline $\mathrm{C} 1-\mathrm{C} 2$ & 1.521 & $\mathrm{C} 3-\mathrm{C} 2^{\mathrm{i}}$ & 1.529 \\
$\mathrm{C} 1-\mathrm{O} 11$ & 1.260 & $\mathrm{C} 3-\mathrm{C} 6$ & 1.556 \\
$\mathrm{C} 1-\mathrm{O} 12$ & 1.270 & $\mathrm{C} 3-\mathrm{O} 17$ & 1.452 \\
$\mathrm{C} 2-\mathrm{C} 3$ & 1.529 & $\mathrm{C} 6-\mathrm{O} 15$ & 1.262 \\
$\mathrm{C} 2-\mathrm{H} 7$ & 1.087 & $\mathrm{C} 6-\mathrm{O} 16$ & 1.261 \\
$\mathrm{C} 2-\mathrm{H} 8$ & 1.092 & $\mathrm{O} 17-\mathrm{H} 18$ & 0.981
\end{tabular}

Symmetry code: (i) $x,-y+3 / 2, z$. 
Hydrogen-bond geometry $\left(A,{ }^{\circ}\right)$

\begin{tabular}{lllll}
\hline$D-\mathrm{H} \cdots A$ & $D-\mathrm{H}$ & $\mathrm{H} \cdots A$ & $D \cdots A$ & $D-\mathrm{H} \cdots A$ \\
\hline $\mathrm{O} 17-\mathrm{H} 18 \cdots \mathrm{O} 16$ & 0.981 & 1.862 & 2.572 & 126.7 \\
\hline
\end{tabular}

